\title{
Inventory Control with Time Delays in Deliveries Using Linear and Quadratic Criteria
}

\author{
Valery I. Smagin, Gennady M. Koshkin, Konstantin S. Kim \\ Institute of Applied Mathematics and Computer Science \\ National Research Tomsk State University \\ Tomsk, Russia \\ e-mail: vsm@mail.tsu.ru,kgm@mail.tsu.ru,kks93@rambler.ru
}

\begin{abstract}
Inventory control algorithm for the model of a warehouse with time delays is proposed. Inventory control algorithm based on optimization linear and quadratic criteria with using the Kalman filtering for systems with unknown input and smoothing procedures is constructed. Example to illustrate the proposed approach is given.
\end{abstract}

Keywords-inventory control; time delays; linear criterion; quadratic criterion; unknown input; Kalman filter; smoothing procedures.

\section{INTRODUCTION}

Synthesis of controls based on optimization both linear and quadratic criteria is applied in many approaches, for example, model predictive control [1], locally optimal control [2]. The main advantage of the method optimal control with such criteria is a significant simplification of the synthesis procedure. These methods have been applied to control in technical systems, chemical processes, inventory control, production-inventory system, and portfolio optimization [3-8].

In this paper, we consider a control algorithm for discrete model of warehouse with time delays. It is assumed that the demand model contains unknown additional terms. In contrast to [7], it is possible to have many delays in the model that means taking into account the deliveries of goods from different suppliers. Also, it is proposed to use the smoothing algorithms for calculating estimates of demand and forecast of demand.

\section{Synthesis OF CONTROLS BY QUADRATIC CRITERION WITH COMPLETE OBSERVATIONS}

Consider the model of the warehouse, which is described by the discrete equation

$$
\begin{gathered}
x(k+1)=A x(k)+\sum_{i=0}^{M} B_{i} u\left(k-h_{i}\right)-s(k) \\
k=0,1,2, \mathrm{~K} ; x(0)=x_{0} ; u(j)=\psi(j), j=-h_{M},-\left(h_{M}-1\right), \mathrm{K},-1 ; \\
h_{M}>h_{M-1}>\mathrm{K}>h_{0} \geq 0
\end{gathered}
$$

where $x(\cdot) \in R^{n}$ is a vector, and $x_{i}$ is a product volume for the $i$-th nomenclature, $u(\cdot) \in R^{m}$ is a vector of deliveries, and $u_{i}(\cdot)$ is a volume of delivery of the $i$-th nomenclature, $h_{i}$ is a time delay, $s(k) \in R^{n}$ is a vector of demand at the $k$-th step $\left(s_{i}(\cdot)\right.$ is a demand for the product of the $i$-th nomenclature), $x_{0}$ and $\psi(j) \quad\left(j=-h_{M},-\left(h_{M}-1\right), \mathrm{K},-1\right)$ are the known vectors. Matrices $A$ and $B_{i}$ define characteristics and the structure of a warehouse.

Take the quadratic criterion in the following form:

$$
\begin{gathered}
I(k)=\mathrm{M}\{x(k+1)-z(k))^{\mathrm{T}} C(x(k+1)-z(k))+ \\
\left.+\sum_{i=0}^{M} u\left(k-h_{i}\right)^{\mathrm{T}} D_{i} u\left(k-h_{i}\right)\right\},
\end{gathered}
$$

where $\mathrm{M}\{\cdot\}$ is the mathematical expectation, $C>0, D_{i} \geq 0$ are weight matrices, $z$ is the tracked vector. In this section, we assume that all the components of the vectors $x$ and $s$ are measured exactly.

Find the optimal control from the equation

$$
\frac{\partial I(k)}{\partial u\left(k-h_{i}\right)}=0 ; i=\overline{0, M}
$$

Taking into account the representation of the model (1) as

$$
x(k)=A^{h_{M}} x\left(k-h_{M}\right)+\sum_{l=1}^{h_{M}} A^{l-1} \sum_{i=0}^{M} B_{i} u\left(k-h_{i}-l\right)-\sum_{l=1}^{h_{M}} A^{l-1} s(k-l)
$$

and condition (3), we obtain the system of equations to find the optimal supplies

$$
\begin{gathered}
u\left(k-\tau_{0}\right)=-\left(B_{0}^{\mathrm{T}} C B_{0}+D_{0}\right)^{-1} B_{0}^{\mathrm{T}} C\left(A^{h_{M}+1} x\left(k-h_{M}\right)+\right. \\
\left.+\sum_{l=1}^{h_{M}} A^{l} \sum_{j=0}^{M} B_{j} u\left(k-h_{j}-l\right)+\sum_{j=1}^{M} B_{j} u\left(k-h_{j}\right)-\sum_{l=1}^{h_{M}} A^{l} s(k-l)-z(k)\right), \\
u\left(k-\tau_{1}\right)=-\left(B_{1}^{\mathrm{T}} C B_{1}+D_{1}\right)^{-1} B_{1}^{\mathrm{T}} C\left(A^{h_{M}+1} x\left(k-h_{M}\right)+\right. \\
\left.+\sum_{l=1}^{h_{M}} A^{l} \sum_{j=0}^{M} B_{j} u\left(k-h_{j}-l\right)+\sum_{\substack{j=0, j \neq 1}}^{M} B_{j} u\left(k-h_{j}\right)+\sum_{l=1}^{h_{M}} A^{l} F s(k-l)-z(k)\right),
\end{gathered}
$$


$\mathrm{M}$

$$
\begin{gathered}
u\left(k-h_{M}\right)=-\left(B_{M}^{\mathrm{T}} C B_{M}+D_{M}\right)^{-1} B_{M}^{\mathrm{T}} C\left(A^{h_{M}+1} x\left(k-h_{M}\right)+\right. \\
+\sum_{l=1}^{h_{M}} A^{l} \sum_{j=0}^{M} B_{j} u\left(k-h_{j}-l\right)+\sum_{j=0}^{M-1} B_{j} u\left(k-h_{j}\right)- \\
\left.-\sum_{l=1}^{h_{M}} A^{l} s(k-l)-z(k)\right) .
\end{gathered}
$$

Since in the system (4) variable $s(\cdot)$ is not always known, there is the problem of its prediction for future time. This prediction may be implemented by various methods of time series forecasting [9-11].

\section{Synthesis OF CONTROLS BY QuAdRATIC CRITERION WITH INCOMPLETE OBSERVATIONS}

Let the model of demand have the form

$$
s(k+1)=R s(k)+f+q(k), s(0)=s_{0},
$$

where $R$ is the known matrix, $f$ is the known vector, $q(k)$ is a random vector. Incomplete observations of the $m_{1}$-vector of demand are defined by the formula

$$
w(k)=H s(k)+\tau(k),
$$

where $H$ is a matrix, $\tau(k)$ is a random vector of errors, $q(k)$ and $\tau(k)$ are sequences of the Gaussian random vectors with the characteristics:

$$
\begin{gathered}
\operatorname{M}\{q(k)\}=0, \operatorname{M}\{\tau(k)\}=0, \quad \operatorname{M}\left\{q(k) q^{T}(j)\right\}=Q \delta_{k j}, \\
\operatorname{M}\left\{\tau(k) \tau^{T}(j)\right\}=T \delta_{k j}, \quad \operatorname{M}\left\{q(k) \tau^{T}(j)\right\}=0,
\end{gathered}
$$

where $\delta_{k j}$ is the Kronecker symbol.

In this case, the control will be calculated according to (4) with making use of the filtering estimates $\hat{s}_{f}(\cdot)$ and the forecasts of demand $\hat{s}_{p}(\cdot)$. For example, to determine $u(0)$, it is necessary to solve system (4) with $k=h_{M}$, which, in this case, is represented in the form:

$$
\begin{gathered}
u\left(h_{M}-h_{0}\right)=-\left(B_{0}^{\mathrm{T}} C B_{0}+D_{0}\right)^{-1} B_{0}^{\mathrm{T}} C\left(A^{h_{M}+1} x(0)+\right. \\
+\sum_{l=1}^{h_{M}} A^{l} \sum_{\substack{j=0 \\
h_{M}-h_{j}-l \geq 0}}^{M} B_{j} u\left(h_{M}-h_{j}-l\right)+\sum_{l=1}^{h_{M}} A^{l} \sum_{\substack{j=0 \\
-h_{M} \leq h_{M}-h_{j}-l<0}}^{M} B_{j} \psi\left(h_{M}-h_{j}-l\right)+ \\
\left.+\sum_{j=1}^{M} B_{j} u\left(\tau_{M}-h_{j}\right)+\sum_{l=1}^{h_{M}-1} A^{l} F \hat{s}_{p}\left(h_{M}-l\right)-A^{h_{M}} \hat{s}_{f}(0)-z\left(h_{M}\right)\right), \\
u\left(h_{M}-h_{1}\right)=-\left(B_{1}^{\mathrm{T}} C B_{1}+D_{1}\right)^{-1} B_{1}^{\mathrm{T}} C\left(A^{h_{M}+1} x(0)+\right. \\
+\sum_{l=1}^{h_{M}} A^{l} \sum_{\substack{j=0 \\
h_{M}-h_{j}-l \geq 0}}^{M} B_{j} u\left(h_{M}-h_{j}-l\right)+\sum_{l=1}^{h_{M}} A^{l} \sum_{\substack{j=0 \\
-h_{M} \leq h_{M}-h_{j}-l<0}}^{M} B_{j} \psi\left(h_{M}-h_{j}-l\right) \\
\left.+\sum_{\substack{j=0, j \neq 1}}^{M} B_{j} u\left(h_{M}-h_{j}\right)-\sum_{l=1}^{h_{M}-1} A^{l} \hat{s}_{p}\left(h_{M}-l\right)-A^{h_{M}} \hat{s}_{f}(0)-z\left(h_{M}\right)\right),
\end{gathered}
$$

$\mathrm{M}$

$$
\begin{gathered}
u(0)=-\left(B_{M}^{\mathrm{T}} C B_{M}+D_{M}\right)^{-1} B_{M}^{\mathrm{T}} C\left(A^{h_{M}+1} x(0)+\right. \\
+\sum_{l=1}^{h_{M}} A^{l} \sum_{\substack{j=0 \\
h_{M}-h_{j}-l \geq 0}}^{M} B_{j} u\left(h_{M}-h_{j}-l\right)+\sum_{l=1}^{h_{M}} A^{l} \sum_{\substack{j=0 \\
-h_{M} \leq h_{M}-h_{j}-l<0}}^{M} B_{j} \psi\left(h_{M}-h_{j}-l\right)+ \\
\left.+\sum_{j=0}^{M-1} B_{j} u\left(h_{M}-h_{j}\right)-\sum_{l=1}^{h_{M}-1} A^{l} s_{p}\left(h_{M}-l\right)-A^{h_{M}} s(0)-z\left(h_{M}\right)\right) .
\end{gathered}
$$

At the next step to find $u(1)$, it is necessary to solve the system (4) with $k=h_{M}+1$, then for $k=h_{M}+2, k=h_{M}+3$, and so on.

The estimates $\hat{s}_{f}(\cdot)$ are the estimates of the optimal Kalman filtering:

$$
\begin{gathered}
\hat{s}_{f}(k)=R \hat{s}_{f}(k-1)+f+K_{f}(k)[w(k), \\
-H\left(R \hat{s}_{f}(k-1)+f\right], \hat{s}_{f}(0)=\bar{s}_{0},
\end{gathered}
$$

where

$$
\begin{aligned}
K_{f}(k)= & P(k / k-1) H^{T}\left(H P(k / k-1) H^{T}+T\right)^{-1} \\
& P(k / k-1)=R P(k-1) R^{T}+Q \\
P(k)= & \left(E_{n}-K_{f}(k) H\right) P(k / k-1), P(0)=P_{0} .
\end{aligned}
$$

In (9), the matrix $E_{n}$ is the identity matrix of the $(n \times n)$ dimension. So, here it is necessary to use extrapolator, which will calculate the estimate of the forecast demand for the 1-st step:

$$
\hat{s}_{p}(k+1)=R \hat{s}_{p}(k)+f+K_{p}(k)\left(w(k)-H \hat{s}_{p}(k)\right), \hat{s}_{p}(0)=\bar{s}_{0},
$$

where

$$
\begin{gathered}
K_{p}(k)=R P_{p}(k) H^{T}\left(H P_{p}(k) H^{T}+T\right)^{-1}, \\
P_{p}(k+1)=\left(R-K_{p}(k) H\right) P_{p}(k)\left(R-K_{p}(k) H\right)^{T} \\
+Q+K_{p}(k) T K_{p}^{T}(k), P_{p}(0)=P_{0} .
\end{gathered}
$$

The forecasts for the next steps $j=2, \ldots, h_{M}-1$ are determined by the formula

$$
\hat{s}_{p}\left(k-h_{M}+j\right)=R \hat{s}_{p}\left(k-h_{M}+j-1\right)+f .
$$

\section{SYNTHESIS OF CONTROLS FOR DEMAND MODEL WITH UNKNOWN PARAMETERS}

Here, we assume that the demand model contains unknown additional parameters:

$$
s(k+1)=(R+\Delta R) s(k)+f+\Delta f+q(k), s(0)=s_{0},
$$

where $R$ is the known matrix, $f$ is the known vector, $\Delta R$ and $\Delta f$ are some addition unknown matrix and vector 
(parameters), which can be interpreted as errors in the model of parameters (12). Model (12) may be interpreted as a dynamic model with an unknown input

$$
s(k+1)=R s(k)+f+r(k)+q(k), s(0)=s_{0},
$$

where $r(k)=\Delta R s(k)+\Delta f$ is an unknown input.

Obtain the filtering estimate on the base of the Kalman filtering algorithm with unknown input $[12,13]$ :

$$
\begin{gathered}
\hat{s}_{f}(k)=R \hat{s}_{f}(k-1)+f+\hat{r}(k-1) \\
+K_{f}(k)\left[w(k)-H\left(R \hat{s}_{f}(k-1)+f+\hat{r}(k-1)\right)\right], \hat{s}_{f}(0)=\bar{s}_{0}, \\
K_{f}(k)=P(k-h / k-1) H^{\mathrm{T}}\left(H P(k-h / k-h-1) H^{\mathrm{T}}+\mathrm{T}\right)^{-1},( \\
P(k / k-1)=R P(k-1) R^{\mathrm{T}}+Q \\
P(k)=\left(E_{n_{1}}-K_{f}(k) H\right) P(k / k-1), P(0)=P_{0}
\end{gathered}
$$

where $\hat{r}(\cdot)$ is given in (24).

The extrapolator, which will estimate the forecast for the 1 -st step $\hat{s}_{p}(k+1)$, we define as follows:

$$
\begin{gathered}
\hat{s}_{p}(k+1)=R \hat{s}_{p}(k)+f+\hat{r}(k)+ \\
+K_{p}(k)\left(w(k)-H \hat{s}_{p}(k)\right), \hat{s}_{p}(0)=\bar{s}_{0}, \\
K_{p}(k)=R P_{p r}(k) H^{\mathrm{T}}\left(H P_{p r}(k) \Phi^{\mathrm{T}}+T\right)^{-1}, \\
P_{p r}(k+1)=\left(R-K_{p}(k) H\right) P_{p r}(k)(R- \\
\left.-K_{p}(k) H\right)^{\mathrm{T}}+Q+K_{p}(k) T K_{p}^{\mathrm{T}}(k), P_{p r}(0)=P_{0} .
\end{gathered}
$$

According to (8), the estimates of forecasts $\hat{s}_{p}(k+j)$ for $j \geq 2$ take the form

$$
\hat{s}_{p}(k+j)=R \hat{s}_{p}(k+j-1)+f+\hat{r}(k+j-1) .
$$

Note that in (29) $\hat{r}(k+j-1)$ for $j \geq 2$ can be calculated using the analysis methods of time series [9-11].

The estimate $\hat{r}$ is calculated by the least square method by criterion [12]

$$
J=\sum_{i=1}^{k}\left\{\|\chi(i)\|_{V}^{2}+\|r(i-1)\|_{W}^{2}\right\},
$$

where $\chi(i)=w(i)-H S(i) \quad(\$(i)=R \hat{s}(i-1)+f) ; V>0, \mathrm{~W} \geq 0$ are weight matrices of the appropriate dimensions, $\|\chi(i)\|_{V}^{2}=\chi^{\mathrm{T}}(i) V \chi(i)$. Then, minimizing (22), we obtain

$$
\hat{r}(k)=\left[H^{\mathrm{T}} V H+W\right]^{-1} H^{\mathrm{T}} V \mathrm{M}\{\Omega(k)\},
$$

where $\Omega(k)=w(k)-H[R \hat{s}(k-1)+f]$.
Now, let us calculate value $\mathrm{M}[\Omega(k)]$ using nonparametric smoothing algorithm $[13,14]$. Applying the analog of the Nadaraya-Watson kernel regression estimates [14], we have

$$
\hat{r}(k)=\left[H^{\mathrm{T}} V H+W\right]^{-1} H^{\mathrm{T}} V \mathrm{M}\{\hat{\Omega}(k)\},
$$

and the $j$-th component of the vector $\hat{\Omega}(k)$ is given as

$$
\hat{\Omega}_{j}(k)=\frac{\sum_{i=1}^{k} \frac{\Omega_{j}(i)}{\mu_{j}} K\left(\frac{k-i+1}{\mu_{j}}\right)}{\sum_{i=1}^{k} \frac{1}{\mu_{j}} K\left(\frac{k-i+1}{\mu_{j}}\right)} .
$$

In the ratio (25), $K(\cdot)$ is a kernel function, $\mu_{j}$ is a bandwidth parameter. We use the Gaussian kernels

$$
K(u)=\frac{\exp \left(\frac{-u^{2}}{2}\right)}{\sqrt{2 \pi}},
$$

and the bandwidths are calculated by the cross-validation method [15].

Note, the estimates (14), (18), and (24) are used by synthesis of controls (7) for system with unknown parameters defined by equations (1) and (13).

\section{Minimization of Expenses Cost}

Define the cost of storage of products in the sliding time interval $[k, k+T]$ using the additional linear criterion

$$
J_{1}(k, z)=\sum_{i=1}^{n} \sum_{t=k}^{k+T} c_{i} x_{i}(t, z)
$$

with the following restrictions:

$$
x_{i}(k) \geq X_{i}, \quad \forall k \in[k, k+T], \quad i=1, \ldots, n,
$$

where $c_{i}$ is a storage cost of product unit for the $i$-th nomenclature in the unit time interval, $X_{i}$ is a safety stock for the $i$-th nomenclature. In (26), the dependence $x_{i}(t, z)$ on $z$ is due control (11) for system (1), (13).

Minimization of the criterion (26) under the restrictions (27) is carried out with respect to the vector $z$ by numerical method, and at every step the control $u(k)$ is recalculated. The obtained value of the optimal vector $z^{*}$ provides the minimum cost in the interval $[k, k+T]$. Vector $z^{*}$ determines the volume of deliveries, and then, by analogy, we solve the problem of minimizing the new criterion $J_{1}(k+1, z)$ under the constraints (27) $(\forall k \in[k+1, k+T+1])$. Such procedure is realized recursively. 


\section{Simulation Results}

Define the data describing the warehouse model and criteria:

$$
\begin{aligned}
& A=\left(\begin{array}{cc}
0.997 & 0 \\
0 & 0.8
\end{array}\right), R=\left(\begin{array}{cc}
0 & 1 \\
0.1 & 0.5
\end{array}\right), f=\left(\begin{array}{l}
0.8 \\
0.8
\end{array}\right) \text {, } \\
& Q=\operatorname{diag}\{0.050 .02\}, h_{0}=1, h_{1}=2, T=\operatorname{diag}\{0.050 .05\} \text {, } \\
& D=W=0, B_{0}=B_{1}=H=C=V=P_{0}=E_{2},
\end{aligned}
$$

Simulations were carried out with the matrix $\Delta R$ and vector $\Delta f$ :

$$
\begin{gathered}
\Delta R=\left(\begin{array}{cc}
0 & 0.02 \\
0.05 & 0.06
\end{array}\right), \\
\Delta f_{1}(k)=\left\{\begin{array}{cc}
0.9, & \text { if } 0 \leq k<50, \\
-0.3, & \text { if } 50 \leq k<100, \\
1.7, & \text { if } 100 \leq k \leq 150,
\end{array}\right. \\
\Delta f_{2}(k)= \begin{cases}1.2, & \text { if } 0 \leq k<50, \\
0.2, & \text { if } 50 \leq k<100, \\
1.7, & \text { if } 100 \leq k \leq 150 .\end{cases}
\end{gathered}
$$

The deliveries are determined for one vehicle under the following restrictions:

$$
\bar{u}_{i}(k)= \begin{cases}(0 \quad 0)^{\mathrm{T}} & \text { if } \quad G(u(k)) \leq G \min , \\ u(k) & \text { if } G \min \leq G(u(k)) \leq G \max , \\ \frac{u(k)}{\alpha(k)} & \text { if } G(u(k)) \geq G \max \end{cases}
$$

where $G \max$ is a vehicle capacity, $G(u(k))=\sum_{i=1}^{2} p_{i} u_{i}(k)$ ( $p_{\mathrm{i}}$ is a weight of product unit of the $i$-th nomenclature), $\alpha(k)=\frac{G(u(k))}{G \max }$ is the compression ratio. The value of $G \min$ is usually defined by the condition

$$
0.8 G \max \leq G \min \leq G \max ,
$$

which provides the high efficient on using of the vehicle.

The simulation results are shown in Fig. 1-7.

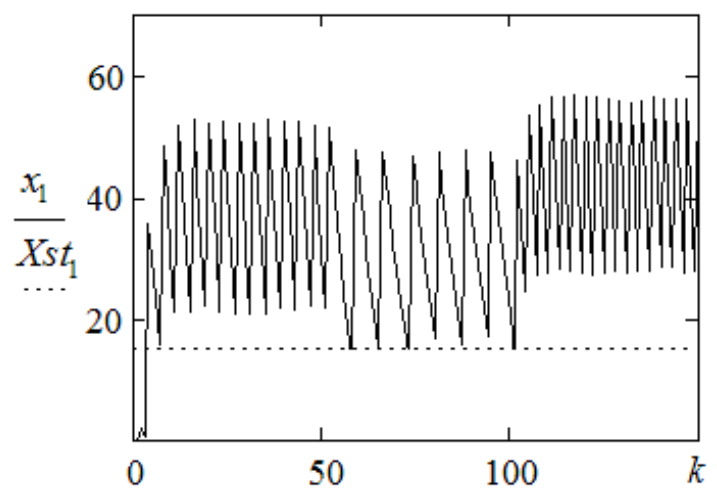

Figure 1. The volume of the stored product demand forecast $\hat{s}_{p, 2}$ for the second nomenclature $x_{1}$ for the first nomenclature

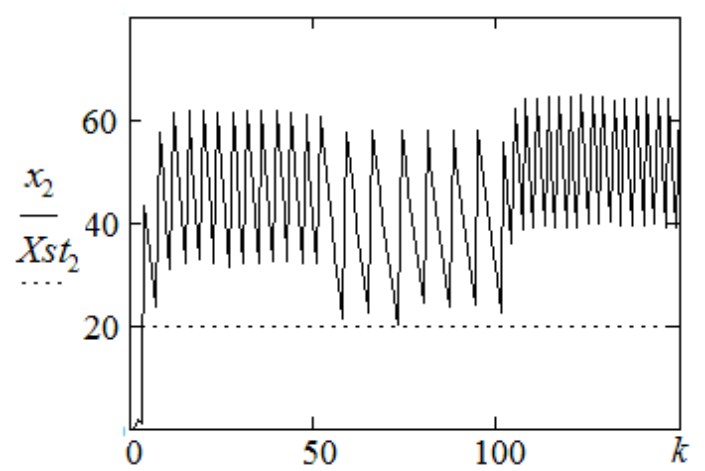

Figure 2. The volume of the stored product $x_{2}$ for the second nomenclature

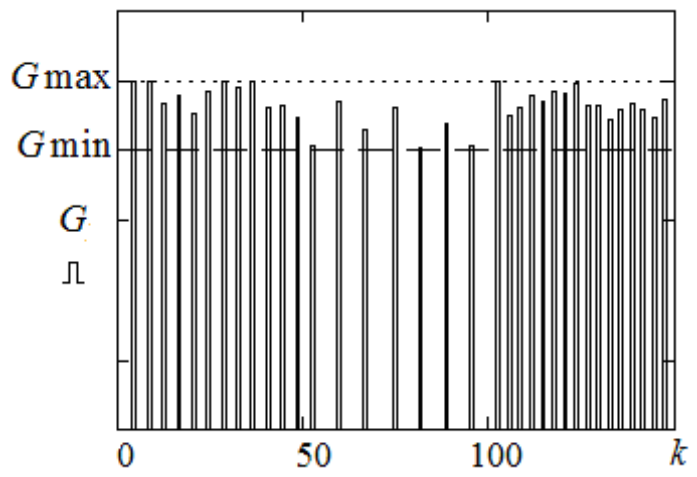

Figure 3. The diagram of the load of the vehicle

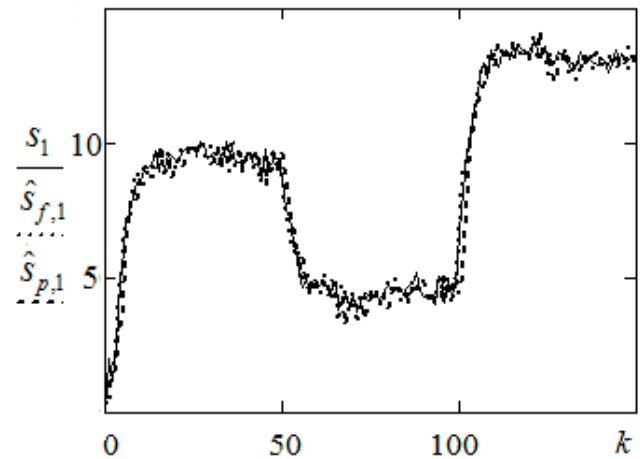

Figure 4. The volume of the demand $s_{1}$, estimate $\hat{s}_{f, 1}$, and demand forecast $\hat{s}_{p, 1}$ for the first nomenclature 


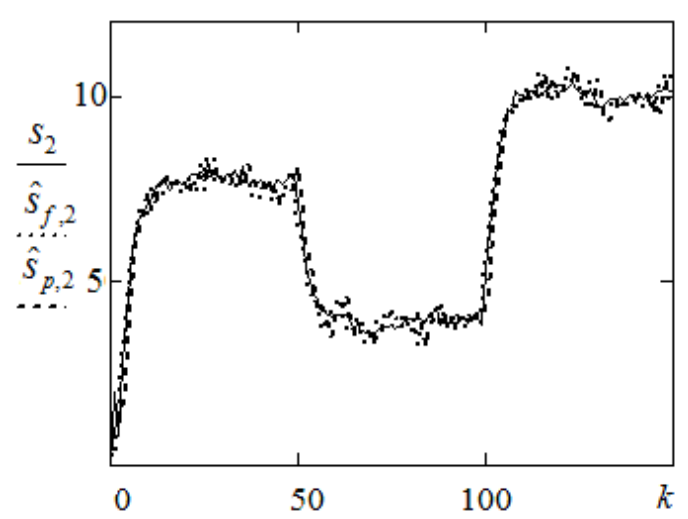

Figure 5. The volume of the demand $s_{2}$, estimate $\hat{s}_{f, 2}$, and

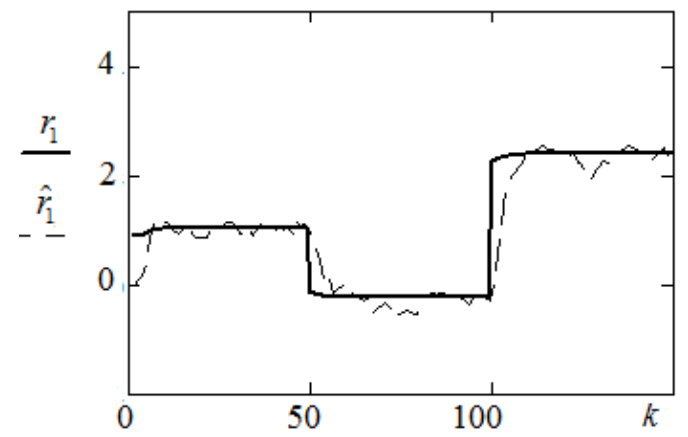

Figure 6. The results of evaluation of unknown input $r_{1}$ in the model of demand

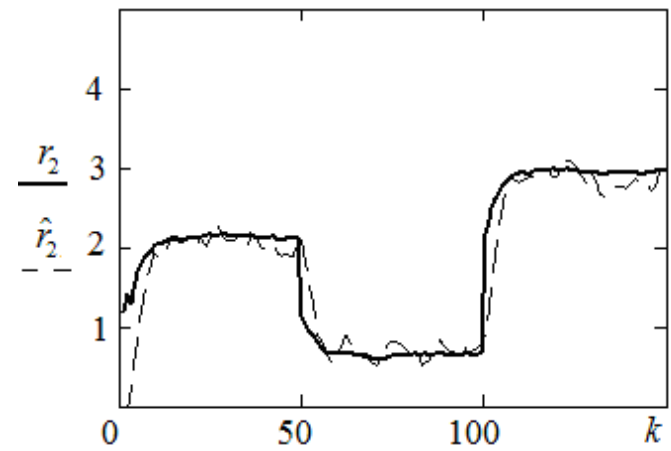

Figure 7. The results of evaluation of unknown input $r_{2}$ in the model of demand

\section{CONCLUSION}

Algorithm of the inventory control with allowance for the vehicle delays from the different supplies for demand models with unknown parameters is developed. It is proposed to use the Kalman filtering algorithms and nonparametric smoothing for calculation of estimate of demand and forecast of demand.

\section{REFERENCES}

[1] E.F. Camacho and C. Bordons, "Model Predictive Control". London: Springer-Verlag, 2004.

[2] M.M. Kogan and Yu.I. Neĭmark, "On the optimality of locally optimal solutions of linear-quadratic problems of control and filtering". Automation and Remote Control, 1992, vol. 53, no. 4, pp.561-569.

[3] P. Conte and P. Pennesi, "Inventory control by model predictive control methods", Proc. 16th IFAC World Congress, Czech Republic, Prague, 2005, pp. 1-6.

[4] E. Aggelogiannaki, Ph. Doganis, and H. Sarimveis, "An adaptive model predictive control conguration for production-inventory systems", Int. J. of Production Economics, 2008, vol. 114, pp. 165-178.

[5] C. Stoica and M. Arahal, "Application of robustied model predictive control to a production-inventory system". Proc. 48th IEEE Conference on Decision and Control and 28th Chinese Control Conference Shanghai, China, 2009, pp. 3993-3998.

[6] J.-C. Henneta, "A globally optimal local inventory control policy for multistage supply chains", Int. J. of Production Research, 2009, vol. 47, issue 2, pp. 435-453.

[7] V.I. Smagin, G.M. Koshkin and R.S. Kim, "Locally Optimal Inventory Control with Time Delay in Deliveries and Incomplete Information on Demand". Proc. II International Symposium on Stochastic Models in Reliability Engineering, Life Science and Operations Management. February 15-18, 2016, Beer Sheva, Israel, pp. 570-574.

[8] V. Dombrovskii and T. Obedko, "Model predictive control for constrained systems with serially correlated stochastic parameters and portfolio optimization". Automatica. 2015. vol. 54, pp. 325-331.

[9] T.W. Anderson, "The Statiatical Analysis of Time Series". New York, John Wiley, 1971.

[10] E. Hannan, "Maltiply Time Series". New York, John Wiley, 1970.

[11] G. Box and G. Jenkins, "Time Series Analysis. Forecasting and Control". San Francisko, Holden Day, 1970.

[12] D. Janczak and Y. Grishin, "State estimation of linear dynamic system with unknown input and uncertain observation using dynamic programming", Control and Cybernetics, vol. 35 , no. 4, pp. 851-862, 2006.

[13] V. Smagin and G. Koshkin, "Kalman filtering and conrol algorithms for systems with unknown disturbances and parameters using nonparametric technique". Proc. $20^{\text {th }}$ Int. Conference on Methods and Models in Automation and Robotics (MMAR), August 2015, Miedzyzdroje, Poland, pp. 247-251.

[14] A. Dobrovidov, G. Koshkin and V. Vasiliev, "Non-parametric state space models", Heber, UT 84032, USA. Kendrick Press, Inc. 2012.

[15] D. Leung, "Cross-validation in nonparametric regression with outliers", Annals of Statistics, vol. 33, pp. 2291-2310, 2005. 